\title{
The Sperm Protein Spaca6 is Essential for Fertilization in Zebrafish
}

\author{
Mirjam I. Binner ${ }^{\dagger}$, Anna Kogan ${ }^{\dagger \neq}$, Karin Panser, Alexander Schleiffer, Victoria E. Deneke ${ }^{\star}$ and \\ Andrea Pauli *
}

Research Institute of Molecular Pathology (IMP), Vienna BioCenter (VBC), Campus-Vienna-Biocenter 1, Vienna, Austria

OPEN ACCESS

Edited by:

Enrica Bianchi,

University of York, United Kingdom

Reviewed by:

Sandrine Barbaux,

Institut National de la Santé et de la Recherche Médicale (INSERM), France

Yuhkoh Satouh,

Gunma University, Japan

Andrew Singson,

Rutgers, The State University of New

Jersey, United States

*Correspondence:

Victoria E. Deneke

victoria.deneke@imp.ac.at

Andrea Pauli

andrea.pauli@imp.ac.at

t'Present address: Anna Kogan,

Department of Biochemistry, University of Oxford, Oxford, United Kingdom

${ }^{\text {F}}$ These authors have contributed equally to this work

Specialty section:

This article was submitted to Molecular and Cellular Reproduction, a section of the journal Frontiers in Cell and Developmental Biology

Received: 01 November 2021 Accepted: 07 December 2021

Published: 03 January 2022

Citation:

Binner MI, Kogan A, Panser K, Schleiffer A, Deneke VE and Pauli A (2022) The Sperm Protein Spaca6 is Essential for Fertilization in Zebrafish.

Front. Cell Dev. Biol. 9:806982. doi: 10.3389/fcell.2021.806982
Fertilization is a key process in all sexually reproducing species, yet the molecular mechanisms that underlie this event remain unclear. To date, only a few proteins have been shown to be essential for sperm-egg binding and fusion in mice, and only some are conserved across vertebrates. One of these conserved, testis-expressed factors is SPACA6, yet its function has not been investigated outside of mammals. Here we show that zebrafish spaca6 encodes for a sperm membrane protein which is essential for fertilization. Zebrafish spaca6 knockout males are sterile. Furthermore, Spaca6deficient sperm have normal morphology, are motile, and can approach the egg, but fail to bind to the egg and therefore cannot complete fertilization. Interestingly, sperm lacking Spaca6 have decreased levels of another essential and conserved sperm fertility factor, Dcst2, revealing a previously unknown dependence of Dcst2 expression on Spaca6. Together, our results show that zebrafish Spaca6 regulates Dcst2 levels and is required for binding between the sperm membrane and the oolemma. This is in contrast to murine sperm lacking SPACA6, which was reported to be able to bind but unable to fuse with oocytes. These findings demonstrate that Spaca6 is essential for zebrafish fertilization and is a conserved sperm factor in vertebrate reproduction.

Keywords: fertilization, zebrafish, sperm-egg interaction, gamete, sperm, reproduction

\section{INTRODUCTION}

Fertilization is an essential process which ensures reproductive success and species survival. Multiple events, such as sperm migration through the female reproductive tract, sperm activation, gamete fusion and egg activation are necessary for fertility and contribute to the formation of a new organism (Bianchi and Wright, 2016; Stein et al., 2020; Deneke and Pauli, 2021). Despite its critical role, little is known about the molecular mechanisms mediating fertilization, particularly sperm-egg interaction and fusion.

In recent years, several proteins have been shown to be essential for sperm-egg interaction in mammals. Most notably, mammalian testis-expressed IZUMO1 and its receptor JUNO on the egg membrane (oolemma) form a complex which is needed for binding of the two gametes prior to fusion (Inoue et al., 2013; Bianchi and Wright, 2016). Izumo1 knockout (KO) sperm can penetrate the zona pellucida (ZP), a glycoprotein coat surrounding the mammalian oocyte, but fail to fuse with the oolemma (Inoue et al., 2005). CD9, an integral membrane protein expressed on the oolemma, has also been shown to be required for gamete fusion (Kaji et al., 2000; le Naour et al., 2000; Miyado et al., 2000). Furthermore, other sperm factors-fertilization influencing membrane protein (FIMP), sperm-oocyte fusion required 1 (SOF1), transmembrane protein 95 (TMEM95), sperm acrosome associated 6 (SPACA6) and the DC-STAMP-like domain-containing proteins DCST1 and 
DCST2-have been shown to be required for sperm-egg fusion in mice (Barbaux et al., 2020; Lamas-Toranzo et al., 2020; Noda et al., 2020; Nado et al., 2021; Inoue et al., 2021). Despite the many factors shown to be necessary for sperm-egg fusion, the combination of these factors appears not to be sufficient to drive fusion in a heterologous system. Noda and others expressed the essential sperm factors involved in fusion, including IZUMO1, in HEK293T cells and observed that HEK293T cells were able to bind but were unable to fuse to ZP-free eggs, indicating the possible need for additional molecules that are involved in this process (Inoue et al., 2013; Noda et al., 2020). Despite these recent advances, the molecular mechanisms of gamete fusion and the interplay between the various sperm and egg factors remain unclear.

Zebrafish has recently emerged as a model organism to study vertebrate fertilization due to its genetic tractability, access to a large number of gametes and external fertilization. The first factor that was discovered to be essential in zebrafish fertilization was Bouncer-a short three-finger-type protein anchored to the egg membrane (Herberg et al., 2018). Bouncer ${ }^{-/}$females are sterile and sperm is unable to bind to Bouncer-deficient eggs, suggesting that Bouncer is involved in sperm-egg membrane binding (Herberg et al., 2018). Interestingly, the mammalian homolog of Bouncer is SPACA4, which is present in sperm and is involved in penetrating the zona pellucida (Fujihara et al., 2021). Moreover, we have shown that DCST1 and DCST2 are also necessary for zebrafish fertilization (Noda et al., 2021). However, while in mice Dcst1 and Dcst $2 \mathrm{KO}$ sperm can bind to, but rarely fuse with oocytes (Inoue et al., 2021; Noda et al., 2021), zebrafish $d c s t 1 / 2 \mathrm{KO}$ sperm already show defects in spermegg binding (Noda et al., 2021). Therefore, zebrafish represents an interesting model system for the study of fertilization; despite the presence of mammalian fertilization factors in fish (Dcst1/2, Bouncer/SPACA4), the currently characterized factors show functional divergence in the fertilization process (Fujihara et al., 2021; Noda et al., 2021). Elucidating the role of conserved fertilization factors in both mammalian and nonmammalian vertebrate species will help to uncover common themes as well as distinct functionalities. Here, we investigate the role of Spaca6 in fertilization in zebrafish.

\section{RESULTS}

\section{Spaca6 is a Conserved Testis-Expressed Membrane Protein}

To study the role of Spaca6 in zebrafish fertilization, we first identified the full-length spaca6 mRNA and protein sequence in zebrafish. Spaca6 has two gene annotations in the most recent zebrafish genome release (GRCz11): a predicted protein-coding gene containing 7 exons (NCBI: XM_021466914.1) and a predicted non-protein coding gene containing 8 exons (ENSEMBL: ENSDART00000155083.2). In addition to the difference in exon numbers, we noticed that the predicted NCBI protein-coding annotation lacked the N-terminus including the signal peptide, which is present in mammalian SPACA6 homologs (Noda et al., 2020). To identify the sequence of the full-length spaca6 transcript that is expressed in testis, we isolated RNA from wild-type zebrafish testes and amplified a 954nt region from cDNA that contained an extended Spaca6 N-terminus and a total of 9 exons. Indeed, coverage tracks derived from RNA sequencing (Herberg et al., 2018; Noda et al., 2021) show expression peaks that align to the mapped transcript as well as specific expression of Spaca6 in testis (Figure 1A, Supplementary Figure S1A), as has been previously reported in mammals (Barbaux et al., 2020; Noda et al., 2020).

The full-length zebrafish spaca6 transcript (GeneID: 101885333; NM_001397778.1) encodes for a 318-amino acid single-pass membrane protein that resembles mammalian SPACA6 proteins in their sequence and predicted tertiary structure (Figures 1B,C). It contains an $\mathrm{N}$-terminal signal peptide, followed by an extracellular region containing an alpha-helical domain (extracellular domain, ECD) and an immunoglobulin (Ig) fold domain, a transmembrane domain and a short cytoplasmic domain (Figure 1B). Alignment of Spaca6 protein sequences from different vertebrates shows that Spaca6 is conserved in its amino acid sequence and secondary structure elements (Figure 1B, Supplementary Figure S1B).

Similar to mouse SPACA6, whose extracellular region has been shown to closely resemble that of IZUMO1 (Nishimura et al., 2016), Alphafold2 (Jumper et al., 2021) predicts the extracellular domain of zebrafish Spaca6 to fold into a fourhelix bundle followed by an Ig-like domain comprised of beta sheets (Figure 1C). In order to taxonomically map Spaca6 homologues, we performed iterative BLASTP searches. This revealed that-similar to Izumo1-Spaca6 homologs are present across vertebrates, while proteins containing a DCSTAMP-like domain, such as Dcst1 and Dcst2, show a much broader distribution across both vertebrates and invertebrates (Figure 1D). Together, the conservation of Spaca6 across vertebrates and predicted similarities in tertiary structure with the mammalian homologs motivated us to assess the functional role of Spaca6 in zebrafish.

\section{Spaca6 is Essential for Male Fertility in Zebrafish}

Using CRISPR/Cas9-mediated mutagenesis, we generated zebrafish lacking Spaca6 protein. To this end, gRNAs were designed to target the third and fourth exons of the full-length spaca6 gene. A mutant harboring an 86-bp deletion was recovered, which resulted in the introduction of a premature stop codon (Figure 2A, Supplementary Figures S1C,D). To test whether Spaca6 is required for fertilization in zebrafish, we assessed the fertilization rates of wild-type, heterozygous and homozygous KO fish (Figures 2B,C). While spaca6 $^{+-}$males had fertilization rates comparable to wild type, spaca6 ${ }^{-1-}$ males were sterile (Figure 2C). Consistent with the expression of Spaca6 exclusively in the male germline (Supplementary Figure S1A), spaca $^{\text {/- }}$ females were fertile (Figure 2C), revealing that Spaca6 is only required for male fertility. To confirm that the observed fertilization defect of spaca $6^{-1-}$ males was caused by the absence of Spaca6 protein, we generated a rescue line that ubiquitously 
A

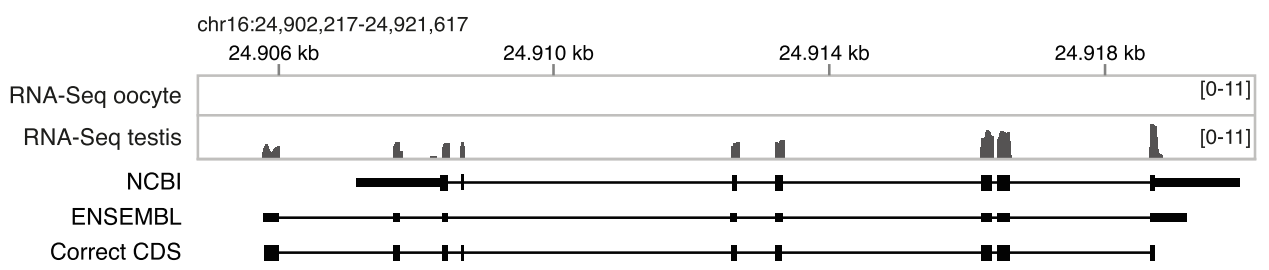

B

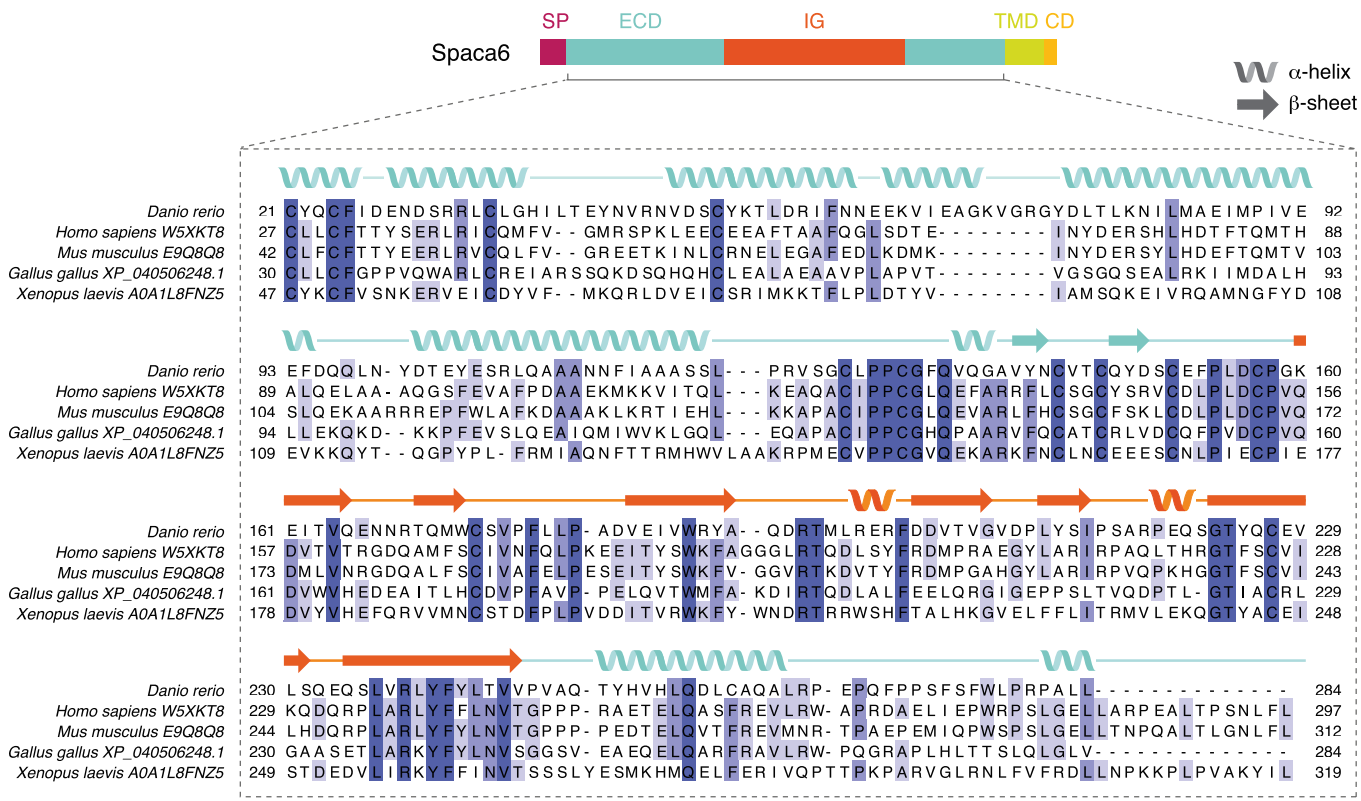

C

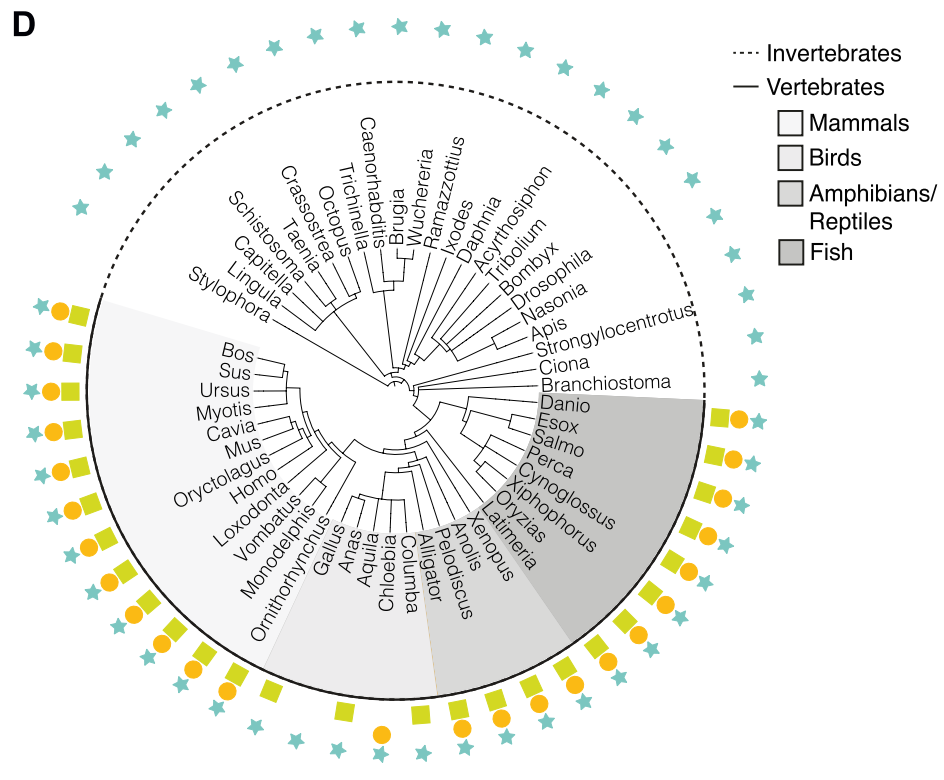

Zebrafish Spaca6 Mouse SPACA6
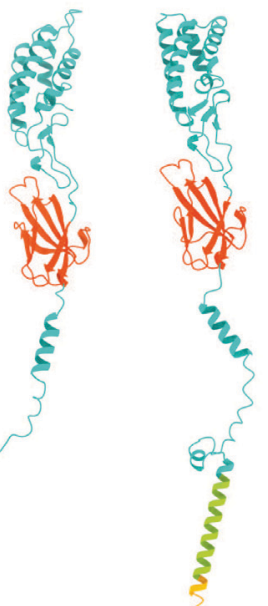

$\downarrow$ DC-STAMP OIzumo1 $\square$ Spaca6

FIGURE 1 | Identification of Spaca6 in zebrafish. (A). Expression and genomic features of zebrafish spaca6. Coverage tracks for RNA sequencing data from oocyte and testis aligned with ENSEMBL (ENSDART00000155083.2) and NCBI (XM_021466914.1) annotations as well as the full-length, newly identified testis coding DNA sequence (correct CDS). Genomic coordinates are based on GRCz11. (B). Protein domain structure of full-length Spaca6 and sequence alignment of the mature extracellular region of selected vertebrate Spaca6 proteins. Secondary structure prediction of the zebrafish Spaca6 protein sequence is depicted above the sequence alignment (coil: alpha-helix; arrow: beta-sheet). The extracellular domain (ECD) is shown in blue, while the Immunoglobulin-like domain (IG) is shown in orange. SP, signal peptide; TMD, Transmembrane domain; CD, Cytoplasmic domain. (C). Tertiary structure predictions of mouse and zebrafish Spaca6. Protein folding 
FIGURE 1 | predictions of mature Spaca6 (lacking the signal peptide sequence) were performed using AlphaFold2 (Jumper et al., 2021 ). Extracellular domain, blue; lglike domain, orange; Transmembrane domain, green; Cytoplasmic domain, yellow. (D). Taxonomic tree of DC-STAMP-like proteins, Izumo1 and Spaca6 across vertebrates and invertebrates. DC-STAMP-like proteins (blue star) are conserved both in vertebrates and invertebrates; Izumo1 (orange circle) and Spaca6 (green square) are conserved only in vertebrates.

A

\begin{tabular}{l} 
genotype \\
\hline wild type \\
spaca6
\end{tabular}

spaca6 ; $\operatorname{tg}[a c t b 2:: s p a c a 6-t 2 a-s f G F P]$
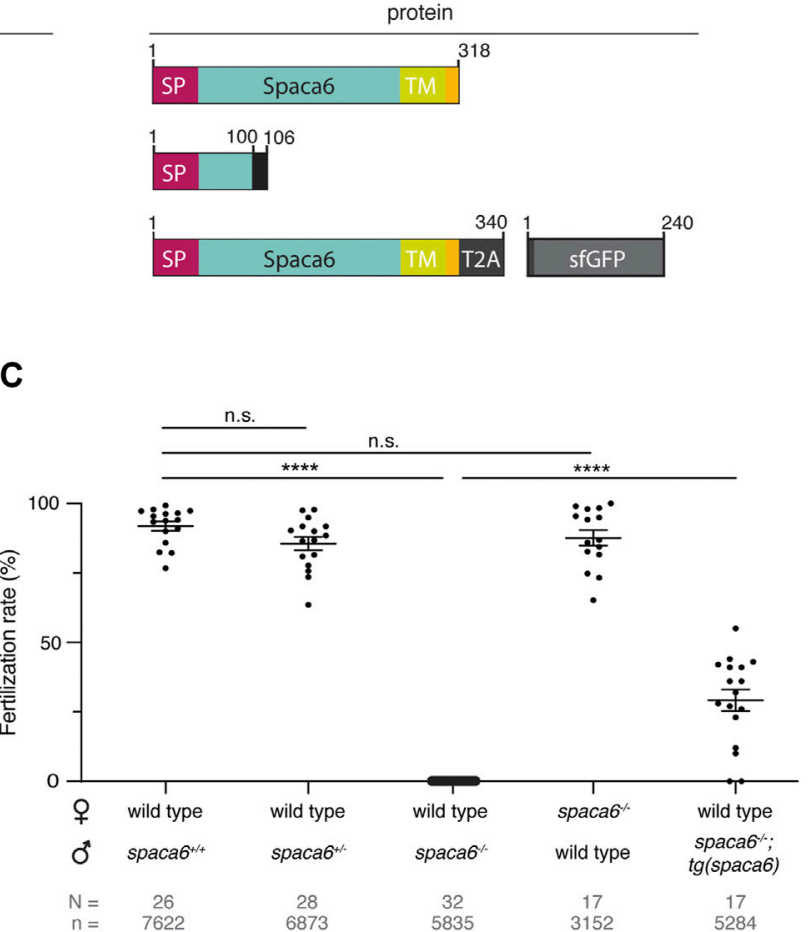

FIGURE 2 |Zebrafish Spaca6 is essential for male fertility. (A). Diagrams of Spaca6 proteins made in wild-type, spaca6 ${ }^{-/-}$and rescue $\left[\right.$spaca6 $^{-/-}$; tg(actb2:spaca6t2a-GFP)] zebrafish lines. An 86-nt deletion in the spaca6 ${ }^{-/-}$line leads to a retained sequence from intron two and a premature stop-codon, resulting in a dysfunctional truncated protein (translated part of the intron is shown in black). In the rescue line, a SGGSG spacer and a part of the viral T2A protein result in a C-terminal 23-aa addition. The amino acid position is given above the scheme. Signal peptide (SP), red; Extracellular domain, blue; Transmembrane domain (TM), green;

Cytoplasmic domain, yellow. (B). Scheme of a mating assay performed to quantify fertilization rates. Fish and gametes are not drawn to scale. (C). Quantification of fertilization rates. Fertilization rates were calculated by counting the number of embryos that developed beyond the one-cell stage. ${ }^{\star \star \star \star}, p<0.0001$ (Mann-Whitney test); n.s, not significant; error bars, standard deviation (SD); $N=$ number of crosses; $n=$ number of embryos.

expresses Spaca6 in a spaca6 ${ }^{-1-}$ background $\left[\right.$ spaca $^{-1}$; $\operatorname{tg}($ actb2: spaca6-T2A-GFP)], hereafter referred to as $\operatorname{spaca6}^{-1}$; $\operatorname{tg}($ spaca6). Transgenic Spaca6 partially rescued the fertilization defect of spaca6 KO males (Figure 2C), suggesting that the fertility defect is indeed due to a lack of Spaca6.

\section{Spaca6 KO Sperm is Motile but Fails to Bind to the Egg Membrane}

To functionally dissect the fertilization defect in spaca6 ${ }^{-/-}$ males, we first assessed whether mutant sperm were morphologically normal. Differential interference contrast (DIC) images did not show any gross morphological differences between spaca6 ${ }^{-/}$and wild-type sperm (Figure 3A). Furthermore, spaca6 $6^{-1-}$ and wild-type sperm had a similar sperm head size and tail length (Figure 3B). In addition, we reasoned that another parameter that may affect sperm fertilizing ability is sperm motility. However, both wild-type and mutant sperm showed comparable motility and directed displacement (Figures 3C,D, Supplementary Movie S1). Therefore, the fertilization defect of spaca6 KO sperm is neither due to morphological defects nor due to an inability of sperm to undergo activation and forward movement.

We next tested whether spaca6 KO sperm is able to approach the micropyle, a funnel-shaped structure within the egg coat that serves as the only sperm entry point in fish (Hart and Donovan, 1983; Wolenski and Hart, 1987; Yanagimachi et al., 2013). In zebrafish, the micropyle measures $30 \mu \mathrm{m}$ in diameter at the surface and gradually tapers off until it reaches the oolemma. This final inner canal is $2.3 \mu \mathrm{m}$ in diameter and is therefore only able to accommodate a single sperm head (Hart and Donovan, 1983; Wolenski \& Hart, 1987). Wild-type and spaca6 $^{-1}$ sperm were added to wild-type eggs and a time-lapse of sperm 
A

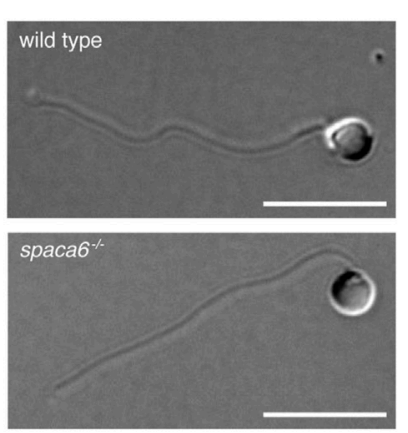

C
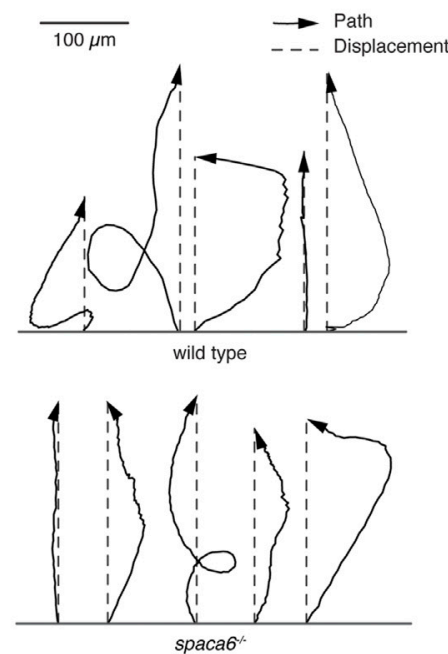

B
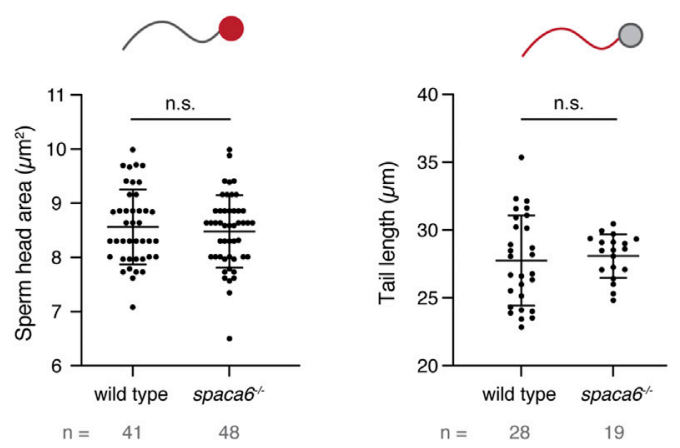

D

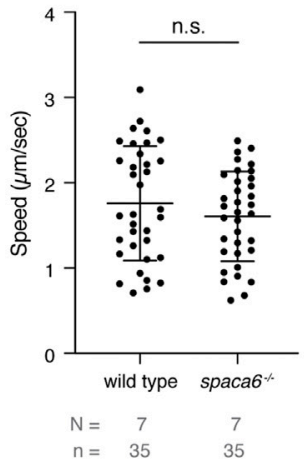

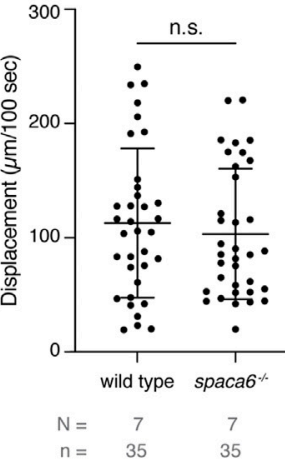

FIGURE 3 | Spaca6 KO sperm is morphologically normal and motile. (A). Representative differential interference contrast (DIC) images of wild-type and spaca6 ${ }^{-/-}$ sperm. Scale bar $=10 \mu \mathrm{m}$. (B). Measurements of the head area (left) and tail length (right) of wild-type and spaca6 $6^{-/-}$sperm. n.s, not significant (Mann-Whitney test, $p>$ 0.05); error bars, SD; $n=$ number of sperm. (C). Representative tracks of wild-type and spaca6 ${ }^{-/}$sperm. Tracks (black arrows) were rotated and aligned at their origin. The relative displacement (grey dashed line) is overlaid. (D). Sperm speed and displacement (per 100 s). n.s, not significant (Mann-Whitney test, $p>0.05$ ); error bars, SD; $N=$ number of independent experiments; $n=$ number of sperm.

approaching the micropyle region was recorded. Both wild-type and mutant sperm were able to reach the micropyle within the same time-frame [number of times sperm successfully approached the micropyle out of the total number of recorded events: 7/9 (wild-type sperm) and 5/6 (spaca6 ${ }^{-1}$ sperm)] (Figure 4A, Supplementary Movie S2). Although spaca6 ${ }^{-1-}$ sperm drifted away after a few minutes (Figure $\mathbf{4 A}$, Supplementary Movie S2), we conclude that spaca6 $^{\text {I- }}$ sperm was able to approach and enter the micropyle similar to wild-type sperm. To test whether spaca6 ${ }^{-1-}$ sperm may be defective in egg binding, we performed a sperm binding assay that we had previously established (Herberg et al., 2018; Noda et al., 2021). This assay reports the ability of sperm to stably adhere to the oolemma, which is a requirement for the subsequent fusion of sperm and egg. In short, wild-type eggs were activated with water and manually dechorionated to expose the entire egg surface. Sperm was then added and sperm binding rates were recorded via time-lapse imaging. As observed in the sperm approach assay, spaca $6^{-1-}$ sperm was able to reach the egg, but failed to stably bind to the egg surface (Figure 4B, Supplementary Movie S3). This was in contrast to wild-type sperm, which was able to stably bind to the egg surface for at least 1 minute (Figure 4C, Supplementary Movie S3). It is worth pointing out that a fraction of the total number of bound wild-type sperm may have not only bound but also fused to the oolemma. While our current assay does not allow us to distinguish bound from potentially already fused sperm, our observation that spaca $\sigma^{1-}$ sperm did not adhere nor showed evidence of prolonged transient interaction with the oolemma indicates that spaca6 ${ }^{-1-}$ sperm is already defective in binding to oolemma. Therefore, we conclude that Spaca6 is required for sperm to stably adhere to the egg plasma membrane in zebrafish.

\section{Dcst2 Levels Are Reduced in spaca6 KO Sperm}

Recent work in mice has shown that SPACA6 levels are decreased in sperm lacking IZUMO1, DCST1 and/or DCST2 (Inoue et al., 2021), suggesting that these essential fertility factors may coregulate each other. To investigate whether this regulation holds 
A

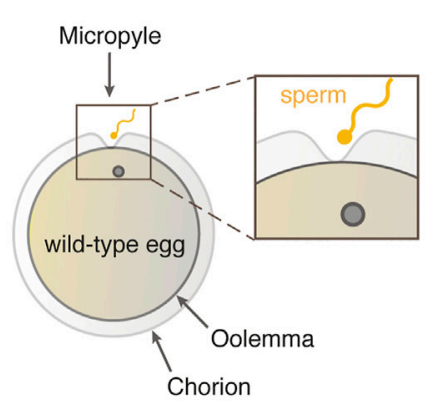

B

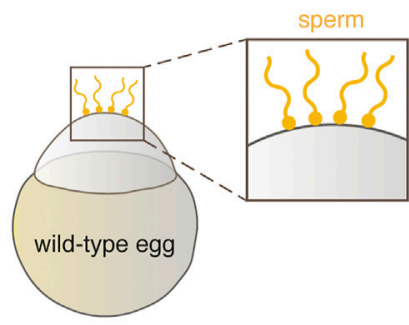

$0 \mathrm{sec}$

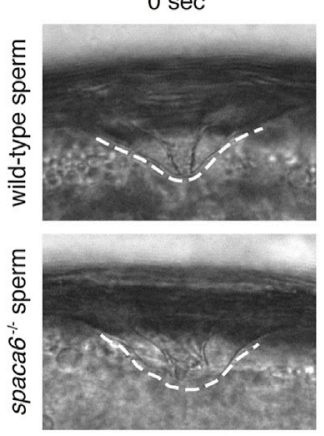

$90 \mathrm{sec}$

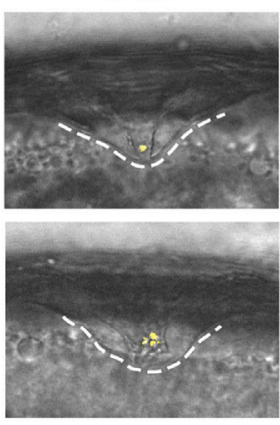

$180 \mathrm{sec}$

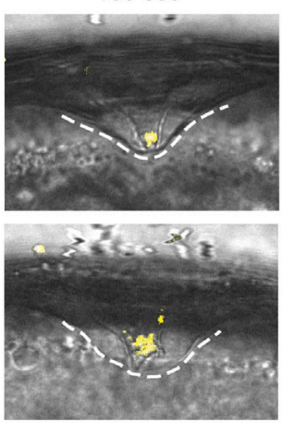

$270 \mathrm{sec}$

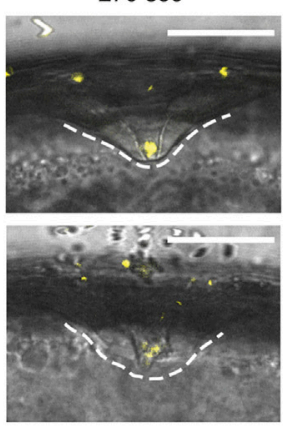

C

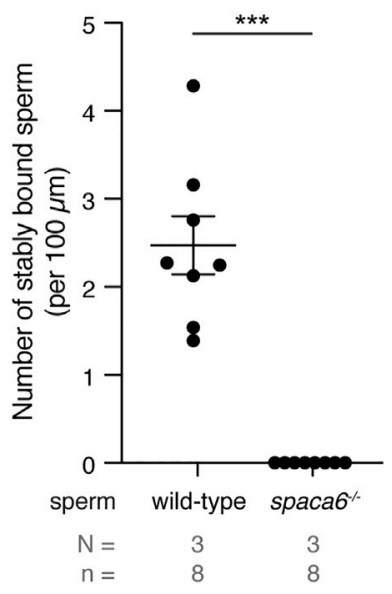

FIGURE 4 |Zebrafish Spaca6 is essential for sperm-egg binding. (A). Time-lapse images of sperm approaching the micropyle of wild-type eggs. Left: Experimental setup. Right: Representative time series of MitoTracker-labeled wild-type (top) and spaca6 ${ }^{-/-}$(bottom) sperm (yellow) approaching the micropyle (white dashed lines). Scale bar $=50 \mu \mathrm{m}$. (B). Sperm binding assay. Left: Experimental setup. Wild-type and spaca6 ${ }^{-/-}$sperm were labeled with MitoTracker (yellow) and subsequently incubated with activated and dechorionated wild-type eggs. Right: Representative images of wild-type (top) and spaca6 ${ }^{-/-}$(bottom) sperm binding to the surface of the egg 2 min after sperm addition. The boxed region is shown at higher magnification below. Scale bar = $100 \mu m$. (C). Quantification of sperm binding. Binding of sperm to the egg was quantified by assessing the number of stably bound sperm per $100 \mu \mathrm{m}$ over a period of 2 min. Wild-type sperm were frequently found bound to the oolemma. ${ }^{* \star *}, p<0.001$ (Mann-Whitney test); error bars, SD; $N=$ number of independent experiments; $n=$ number of sperm.

true reciprocally, we measured Dcst2 protein levels and localization in spaca $^{-1-}$ sperm, using an antibody recognizing zebrafish Dcst2 (Noda et al., 2021). Dcst2 levels were significantly decreased in spaca $^{-1-}$ sperm, as revealed by immunoblotting (Figures 5A,B, Supplementary Figure S2) and immunofluorescence imaging, which showed a reduction of Dcst2-positive foci around the sperm plasma membrane (Figure 5C). The decrease in Dcst2 levels was partially restored in sperm of the spaca6 $^{-/} ; \operatorname{tg}($ spaca6) rescue line, which is consistent with the partial rescue of fertility (Figure 2B). Our data therefore suggests that Spaca6 regulates Dcst2 protein levels.

\section{DISCUSSION}

Our results show that testis-expressed Spaca6 is essential for zebrafish fertilization. We make two major findings: 1) In zebrafish, the absence of Spaca6 leads to a disruption in the sperm's binding ability to the egg surface and 2) the levels of another key fertilization factor, Dcst2, depend on the presence of Spaca6.

Our study shows that Spaca6 has an essential role in fertilization outside of mammals. Even though Spaca6 is conserved among vertebrates (Figures 1B-D), the step at which fertilization stalls in the absence of Spaca6 differs between zebrafish and mice. Mouse Spaca6 KO sperm can penetrate the zona pellucida and bind to the oolemma, but fail to fuse with the egg (Barbaux et al., 2020; Noda et al., 2020). In contrast, our findings in zebrafish reveal that zebrafish sperm lacking Spaca6 is already unable to bind to the egg membrane, and is thus required in a step before fusion can take place.

On a molecular level the observed difference could possibly be reconciled from mouse experiments involving IZUMO1. One possibility is that mouse Spaca6 KO sperm is still able to bind to the oocyte due to the presence of IZUMO1, thereby providing a redundant role in sperm adhesion to the egg. In support of this notion, IZUMO1 protein levels as well as IZUMO1 relocation 

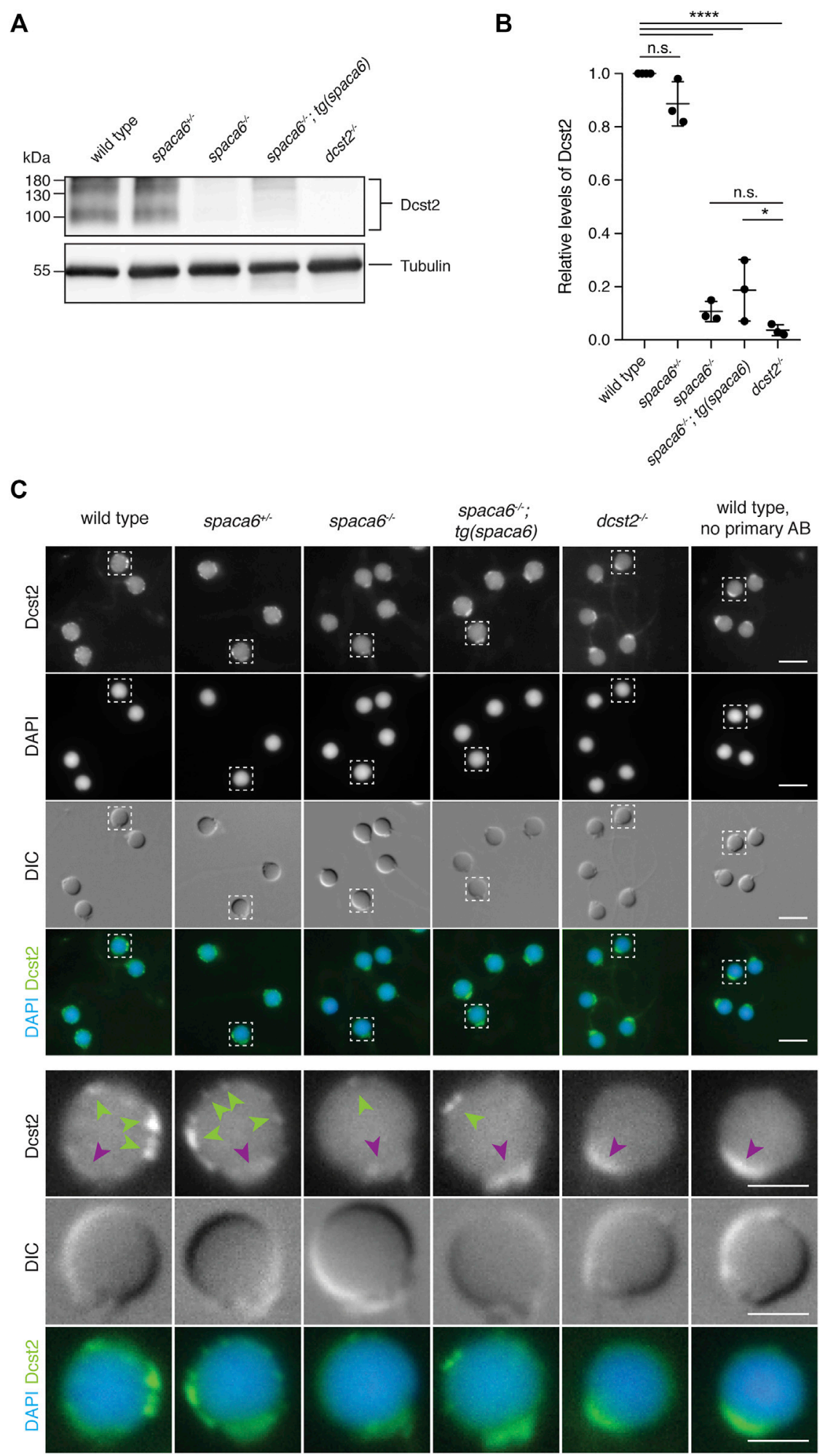

FIGURE 5 | Dcst2 levels are reduced in zebrafish spaca6 KO sperm. (A). Immunoblot of sperm samples of different genotypes probed with antibodies against zebrafish Dcst2. Zebrafish Dcst2 is predicted to be a glycoprotein, resulting in a wider area of detected signal. Tubulin protein levels of the same blot are shown as loading control. The uncropped immunoblot is shown in Supplementary Figure S2. (B). Quantification of sperm Dcst2 levels of different genotypes based on three independent immunoblots. Values were compared to tubulin levels and normalized to wild type. Statistical significance was calculated using one-way ANOVA and multiple comparisons analysis: n.s, not significant $\left(p>0.05\right.$; $\left.{ }^{\star \star \star \star}, p<0.0001 ;{ }^{*}, p=0.045\right)$. (C). Immunofluorescent images of Dcst2 protein in sperm heads of different 
FIGURE 5 | genotypes. Upper panels: Overview images (scale bar $=5 \mu \mathrm{m})$. Lower panels: Higher magnification images of an individual exemplary sperm head (boxed in the upper panels) for each genotype (scale bar $=2 \mu \mathrm{m}$ ). Sperm were stained with DAPI (blue) to visualize nuclei and antibodies against zebrafish Dcst2 (green). Dcst2 localizes to distinct foci around the sperm head membrane (green arrowheads in the lower panels) in wild-type, spaca6 ${ }^{+/}$and tg(spaca6)-rescued sperm.

Autofluorescence of the sperm midpiece appears as a uniform signal in the Dcst2 channel (magenta arrowhead).

during the acrosome reaction were reported to be normal in Spaca6 KO sperm (Barbaux et al., 2020; Inoue et al., 2021). Moreover, IZUMO1 was shown to be sufficient for cells to bind to the oocyte (Inoue et al., 2013; Noda et al., 2020), suggesting that one major role of IZUMO1 is to enable sperm to adhere to the egg surface. Although zebrafish have an Izumo1 ortholog (Figure 1C), its mammalian binding partner on the egg surface, JUNO, appears to be absent in fish (Grayson, 2015). Izumol's presence may therefore not be sufficient to mediate binding between the two gametes in fish. Future experiments exploring the role of Izumol in zebrafish fertilization as well as potential co-regulation of Spaca6 and Izumol will help elucidate the molecular mechanisms in fish and mammalian systems alike.

Direct evidence that essential sperm factors co-regulate each other was recently reported in mice (Inoue et al., 2021). Inoue and others showed that sperm lacking IZUMO1, DCST1 and/or DCST2 had undetectable levels of SPACA6 protein, which suggested that SPACA6 levels are dependent on the presence of each of these factors. Interestingly, SPACA6 protein levels were restored when IZUMO1 was transgenically expressed in Izumo1 KO sperm, but not when DCST1/2 were expressed in Dcst1/2 KO sperm, even though fertilization was rescued. One possibility is that SPACA6 levels were undetectable in the rescue but high enough for fertilization to be restored. Another possibility is that the role of SPACA6 can be by-passed by transgenic expression of DCST1/2, suggesting an indirect role of SPACA6 in fertilization. Our data shows that in zebrafish, transgenic expression of Spaca6 results in a partial rescue of fertilization (Figure 2C), which is correlated with a partial restoration of Dcst2 levels (Figure 5). However, it is currently unclear whether this partial rescue in fertilization is due to insufficient levels of Spaca6, Dcst1/2 or both. Together, this study and studies in mice point towards a coregulation of SPACA6 and DCST1/2. Inoue et al. demonstrated that DCST1/2 regulates the levels of SPACA6 (Inoue et al., 2021), and we show that zebrafish Dcst2 levels depend on the presence of Spaca6 (Figure 5). Whether these factors act purely as stabilization factors or whether they also play a role in spermegg interaction remains to be tested. Further studies characterizing the molecular co-regulation of these factors will be necessary to understand their role in protein stability, binding and fusion.

In conclusion, Spaca6 is a conserved factor essential for fertilization in vertebrates, but its molecular function still remains unclear. Judging from the current data, there are several possible explanations to reconcile the role of Spaca6. Since Dcst2 levels are disrupted in spaca6 KO sperm in zebrafish, Spaca6 may serve as a stabilization factor. While this idea has not yet been tested experimentally in mammals, loss of DCST1/2 protein levels in mammalian Spaca6 KO sperm would provide direct evidence for a conserved role of Spaca6 across vertebrates in stabilizing Dcst1/2. Alternatively, due to Spaca6's structural similarity to Izumo1 (Nishimura et al., 2016) - a wellknown adhesion factor - and the inability of spaca6 KO sperm to bind to the egg surface in zebrafish, Spaca6 could be involved in sperm-egg adhesion, which may or may not depend on its regulation of Dcst1/2. In this context, studies of its potential interaction partner(s) on the egg membrane in zebrafish and mice might identify new fertilization factors on the egg. Finally, the notion of a membrane complex needed for binding and fusion has been previously proposed (Barbaux et al., 2020; Noda et al., 2020). Spaca6 may contribute to forming and/or stabilizing such a multi-factor complex on the sperm membrane that regulates both binding and fusion. Further investigation of the co-regulation and potential interaction between Spaca6, Dcst1/2, Izumo1 and other known essential fertilization factors may help elucidate the mechanism of gamete fusion on a molecular level.

\section{MATERIALS AND METHODS}

\section{Zebrafish Husbandry}

Zebrafish (Danio rerio) were raised according to standard protocols $\left(28^{\circ} \mathrm{C}\right.$ water temperature, $14 / 10 \mathrm{~h}$ light/dark cycle). $\mathrm{TLAB}$ fish were generated by crossing $\mathrm{AB}$ and natural variant TL (Tupfel Longfin) zebrafish and used as wild type for all experiments. The generation of zebrafish spaca6 $\mathrm{KO}$ fish is described below. The $d c s t 2^{-/-}$zebrafish line has been described previously (Noda et al., 2021). All fish experiments were conducted according to Austrian and European guidelines for animal research and approved by the Amt der Wiener Landesregierung, Magistratsabteilung 58-Wasserrecht.

\section{Identification of the Full-Length Zebrafish Spaca6 Sequence}

Current gene annotations for zebrafish spaca6 (NCBI Danio rerio Annotation release 106: XM_021466914.1, seven exons; and ENSEMBL release 104: BX539313.2-201, ENSDART00000155083.2, eight exons) were found in the Genome Browser (http://genome.ucsc.edu) using the zebrafish genome release (GRCz11). To obtain the correct, full-length sequence for zebrafish Spaca6, wild-type zebrafish testis cDNA was used for amplifying a region predicted to encompass the fulllength protein sequence (primers used for PCR: Spaca6_CDS_F: GCTACTTGTTCTTTTTGCAGGATCCGCCACCATGTTTGT GTTTATTGCAAAAC and Spaca6_CDS_R: ACACTCCTGATC CTCCTGAGAATTCGGCTGGATTAGAAACGTTG). The amplified region was cloned and subsequently sequenceverified (submitted to NCBI as GeneID:101885333; NM_ 001397778.1). Published RNA sequencing data from adult tissues (Herberg et al., 2018; Noda et al., 2021) was used to analyse spaca6 gene expression levels in various adult tissues and 
to examine the coverage tracks across spaca6 in testis and oocytes using the Integrative Genomics Viewer (IGV) (http://software. broadinstitute.org/software/igv/).

\section{Taxonomic Tree of Spaca6, Izumo1 and DC-STAMP-like Proteins and Analysis of Spaca6 Protein Structure and Conservation}

Spaca6 orthologs were collected in a series of NCBI blast searches starting with human SPACA6 (sp|W5XKT8|SACA6_HUMAN) and zebrafish Spaca6 (ref|XP_021322589.1|) from the UniProt reference proteomes or NCBI non redundant (nr) protein database applying significant E-value thresholds (1e-05) (Altschul, 1997; Schoch et al., 2020; Bateman et al., 2021). Sequences of the DC-STAMP protein family, including human DCST1, DCST2, DC-STAMP, and OC-STAMP, were identified in a Hidden Markov Model (HMM) search using the PFAM DCSTAMP model against UniProt reference proteomes databases applying significant E-value thresholds $(<0.01)$ (Eddy, 1998; ElGebali et al., 2019). Izumo1 protein family members were identified using the PFAM IZUMO HMM search tool, covering the amino-terminal conserved region of human IZUMO1 (21-165, sp|Q8IYV9|IZUM1_HUMAN) in the UniProt reference proteomes databases (E-value $<0.01$ ). In addition, an extended region of IZUMO1 (corresponding to human 1-219) was used and searched for with NCBI blastp in the NCBI nr database (E-value < 0.001). Out of the full set of 453 taxa containing either DC-STAMP, Izumol or Spaca6 proteins, 52 representative animal species were selected, and a taxonomic tree was retrieved using the NCBI Taxonomy CommonTree tool (Schoch et al., 2020). The tree visualization was performed in iTOL v6 (Letunic and Bork, 2021).

The protein sequence alignment of vertebrate Spaca6 amino acid sequences was performed using the Muscle alignment tool (http://www.drive5.com/muscle/, version 3.8.31) and visualized with Jalview (Waterhouse et al., 2009). The percentage of sequence identity of the mature Spaca6 protein of different vertebrate species was derived using the Percent Identity Matrix from Clustal Omega (Sievers et al., 2011).

The protein domain predictions for zebrafish Spaca6 and mouse SPACA6 (Uniprot ID: E9Q8Q8) were obtained from InterPro (Blum et al., 2021). Additionally, the Ig-like domain annotation was derived from previously published data for mouse IZUMO1 and SPACA6 (Nishimura et al., 2016). Secondary and tertiary protein structure predictions were obtained using AlphaFold2 (Jumper et al., 2021). The mouse SPACA6 model (Identifier: AF-E9Q8Q8-F) was already predicted, whilst the zebrafish Spaca6 tertiary structure was modeled using the newly identified Spaca6 amino acid sequence without the signal peptide.

\section{Generation of spaca6 KO Zebrafish}

Spaca6 KO fish were generated by Cas9-mediated mutagenesis. Two guide RNAs (sgRNAs) targeting the third and fourth exons of the full-length spaca6 gene were generated according to published protocols (Gagnon et al., 2014) by oligo annealing followed by $\mathrm{T} 7$ polymerase-driven in vitro transcription (gene- specific targeting oligos: spaca6_1_gRNA: TAATACGACTCA CTATAGGCGGCCTCAAGCCTGCCCAGGTTTTAGAGC

TAGAAATAGCAAG and spaca6_2_gRNA: TAATACGACTCA CTATAGGTCTGGATGTTTGCCCCCATGGTTTTAGAGCTA GAAATAGCAAG; common tracer oligo AAAAGCACCGAC TCGGTGCCACTTTTTCAAGTTGATAACGGACTAGCCTTA TTTTAACTTGCTATTTCTAGCTCTAAAAC). Cas9 protein and spaca6 sgRNAs were co-injected into the cell of one-cell stage TLAB embryos. Putative founder fish were outcrossed to TLAB wild-type fish. A founder fish carrying a germline mutation in the spaca6 gene was identified by a size difference in the spaca6 PCR amplicon in a pool of embryo progeny (primers: spaca6_gt_F: GCAGAGAAATCTTGATTG GAGG and spaca6_gt_R: AAGCAGACCAGTATACAATTT TTGC). Embryos from this founder fish were raised to adulthood. Amplicon sequencing of adult fin-clips identified the 86-nt deletion, which results in a frameshift mutation and a premature stop codon in intron 2 (GRCz11: Chr16:24,907,548). Genotyping of spaca6 mutant fish was performed by PCR using primers: spaca6_gt_F and spaca6_gt_R. Detection of the deletion was performed by standard gel electrophoresis using a $4 \%$ agarose gel. Homozygous spaca $6^{-1-}$ fish were generated by outcrossing spaca $6^{+/-}$fish to wild-type fish and then incrossing spaca $^{+/-}$fish from the next generation.

\section{Generation of Zebrafish Expressing Transgenic Spaca6}

The full-length spaca6 coding sequence, including the spaca6 signal peptide, the extracellular region and transmembrane and intracellular domains, was amplified by PCR from cDNA derived from adult zebrafish testis (Spaca6_CDS_F and Spaca6_CDS_R) and subcloned by Gibson cloning (Gibson et al., 2009) into a vector for Tol2-mediated transgenesis along with a SG-linkerT2A-sfGFP sequence inserted in frame immediately before the stop-codon of the spaca6 sequence (resulting vector: pMTB Tol2 actb2-promoter-spaca6-SG-linker-T2A-sfGFP SV40UTR). Zebrafish lines expressing transgenic Spaca6 were generated by injecting the spaca6 expression construct with Tol2 mRNA into spaca $^{+/-}$zebrafish embryos $(15 \mathrm{ng} / \mu \mathrm{l}$ of the plasmid in RNasefree water, $35 \mathrm{ng} / \mu \mathrm{l}$ Tol 2 mRNA, $0.083 \%$ phenol red solution [Sigma-Aldrich]), following standard procedures. Injected embryos with high expression of sfGFP at 1 day post fertilization were raised to adulthood. Putative founder fish were crossed to spaca $^{\text {/- }}$ or spaca6 $6^{+-}$fish and the progeny was screened for fluorescence, raised to adulthood and genotyped using primers spaca6_gt_F and spaca6_gt_R to identify adult fish lacking endogenous spaca6. Spaca6 ${ }^{-1}$ male fish expressing transgenic spaca6 $\left[\right.$ spaca $^{/-} ; \operatorname{tg}($ spaca6) $]$ were crossed to wildtype females in order to quantify fertilization rates and assess functionality of the construct.

\section{Quantification of in vivo Fertilization Rates in Zebrafish}

The evening prior to mating, male and female fish were separated in breeding cages (one male and one female per cage). The next 
morning, male and female fish were allowed to mate. Eggs were collected and kept at $28^{\circ} \mathrm{C}$ in $\mathrm{E} 3$ medium $(5 \mathrm{mM} \mathrm{NaCl}, 0.17 \mathrm{mM}$ $\mathrm{KCl}, 0.33 \mathrm{mM} \mathrm{CaCl}_{2}, 0.33 \mathrm{mM} \mathrm{MgSO}_{4}, 0.00001 \%$ Methylene blue). The rate of fertilization was assessed approximately $3 \mathrm{~h}$ post laying. By this time, fertilized embryos have developed to $\sim 1000$-cell stage embryos, while unfertilized eggs resemble onecell stage embryos. Direct comparisons were made between siblings of different genotypes [wild type; spaca $^{/-}$; spaca6 ${ }^{+- \text {; }}$ spaca6 $^{-1-} \operatorname{tg}($ actb2:spaca6-t2a-GFP $\left.)\right]$.

\section{Assessment of Sperm Morphology}

To collect wild-type or mutant sperm, male zebrafish were anesthetized using $0.1 \%$ tricaine. Sperm was collected with a glass capillary from the urogenital opening and immediately fixed with $3.7 \%$ formaldehyde at $4^{\circ} \mathrm{C}$ for $20 \mathrm{~min}$. Sperm were spun onto an adhesive slide using a CytoSpin 4 (Thermo Fisher Scientific) at $1,000 \mathrm{rpm}$ for $5 \mathrm{~min}$ followed by permeabilization with ice-cold methanol for $5 \mathrm{~min}$ and a wash with $0.1 \%$ Tween in $1 \mathrm{x}$ PBS (PBST). After mounting using VECTASHIELD Antifade with DAPI (Vector Laboratories), sperm were imaged with an Axio Imager.Z2 microscope (Zeiss) with an oil immersion $63 \mathrm{x} /$ 1.4 Plan-Apochromat DIC objective.

\section{Assessment of Sperm Motility}

Sperm were isolated from 1 to 2 wild-type and mutant male fish and kept in $100 \mu \mathrm{l}$ Hank's saline $(0.137 \mathrm{M} \mathrm{NaCl}, 5.4 \mathrm{mM} \mathrm{KCl}$, $0.25 \mathrm{mM} \mathrm{Na}_{2} \mathrm{HPO}_{4}, 1.3 \mathrm{mM} \mathrm{CaCl}_{2}, 1 \mathrm{mM} \mathrm{MgSO}$, and $4.2 \mathrm{mM}$ $\mathrm{NaHCO}_{3}$ ) containing $0.5 \mu \mathrm{M}$ MitoTracker Deep Red FM (Molecular Probes) for $>10 \mathrm{~min}$ on ice. Sperm (approximately $5,000 \mathrm{sperm} / \mu \mathrm{l}$ ) were activated using E3 medium in a 1:4 dilution and placed onto a $10 \mu \mathrm{m}$ thick chamber slide (Leja counting chamber, SC 10-01-04-B). Sperm motility was imaged $30 \mathrm{~s}$ after activation using an Axio Imager.Z2 microscope (Zeiss) and a 10x/ 0.3 plan-neofluar objective using darkfield. Sperm tracks were analyzed using Fiji with the "manual tracking" plugin (Cordelieres, 2005). Sperm that were present in the movie for more than 30 timeframes were tracked for as many frames as possible. Coordinates of the sperm cells were used to calculate average sperm speed and displacement. Sperm displacement was calculated by measuring the distance between the first and the last coordinates (normalized by 100 timeframes).

\section{Imaging of Zebrafish Sperm Approach}

Sperm were isolated from 2 to 4 wild-type and mutant male fish and kept in $150 \mu \mathrm{l}$ Hank's saline containing $0.5 \mu \mathrm{M}$ MitoTracker Deep Red FM (Molecular Probes) for $>10 \mathrm{~min}$ on ice. Unactivated, mature eggs were then isolated from a wild-type female. To prevent activation, eggs were kept in sorting medium (Leibovitz's medium, 0.5\% BSA, $\mathrm{pH}$ 9.0) at room temperature. Eggs were kept in place using a petri dish with cone-shaped agarose molds (1.5\% agarose in sorting medium) filled with sorting medium. Imaging was performed with a LSM800 Examiner Z1 upright system (Zeiss) with a 20x/ 1.0 plan-apochromat water dipping objective. Before sperm addition, sorting media was removed and $1 \mathrm{ml}$ of E3 medium carefully added close to the egg. $3 \mu$ of stained spermatozoa (approximately 150,000-300,000 sperm) was added as close to the egg as possible during image acquisition. The resulting timelapse movies were analyzed using Fiji. Timestamps were calculated beginning with the addition of sperm to the eggs.

\section{Imaging and Analysis of Zebrafish Sperm-Egg Binding}

Sperm were isolated from 2 to 4 wild-type and mutant male fish and kept in $200 \mu$ l Hank's saline containing $0.5 \mu \mathrm{M}$ MitoTracker Deep Red FM (Molecular Probes) on ice. Unactivated, mature eggs were squeezed from a wild-type female fish and activated by addition of E3 medium. After $10 \mathrm{~min}, 1-2$ eggs were manually dechorionated using forceps and one egg was transferred to a cone-shaped $2 \%$ agarose-coated imaging dish with E3 medium. After focusing on the egg plasma membrane, the objective was briefly lifted to add $2-5 \mu \mathrm{l}$ of stained sperm (approximately 200,000-250,000 sperm). Imaging was performed with a LSM800 Examiner Z1 upright system (Zeiss) using a $20 \mathrm{x} / 0.3$ Achroplan water dipping objective. Images were acquired until sperm were no longer motile $(5 \mathrm{~min})$. To analyze sperm-egg binding, stably bound sperm were counted. Sperm were counted as bound when they remained in the same position for at least 2 min following a 90-s period in which they activated and approached the egg. Data was plotted as the number of sperm bound per $100 \mu \mathrm{m}$ of egg membrane for 2 minutes.

\section{Sperm Immunocytochemistry}

Sperm from zebrafish males was collected in 3.7\% formaldehyde diluted in Hank's saline solution and stored on ice for $20 \mathrm{~min}$ to $1 \mathrm{~h}$. Sperm was pelleted by centrifugation at $850 \mathrm{rpm}$ for $3 \mathrm{~min}$, and the fixative was replaced with Hank's saline. Sperm was spun onto an adhesive slide with a CytoSpin 4 (Thermo Fisher Scientific) at $800 \mathrm{rpm}$ for $3 \mathrm{~min}$. Slides were briefly washed once in 1x PBS, and the sperm was permeabilized in $0.25 \%$ Tween in $1 \mathrm{x}$ PBS for $30 \mathrm{~min}$ before blocking with 10\% normal goat serum (Invitrogen) and $40 \mu \mathrm{g} / \mathrm{ml} \mathrm{BSA}$ in PBST for at least $1 \mathrm{~h}$. Slides were then incubated with mouse anti-zebrafish-Dcst2 antibody in blocking buffer (1:650; (Noda et al., 2021)) overnight at $4^{\circ} \mathrm{C}$ in a humidified chamber. After several washes with PBST, slides were incubated with goat antimouse IgG Alexa Fluor 488 secondary antibody (1:380, Thermo Fisher Scientific) for $1 \mathrm{~h}$, washed several times with PBST and finally once with 1x PBS. After mounting using VECTASHIELD Antifade with DAPI (Vector Laboratories), sperm was imaged with an Axio Imager.Z2 microscope (Zeiss) using an oil immersion 100x/ 1.4 plan-apochromat objective. Widefield sperm images were processed for each genotype using Fiji by adjusting image brightness and contrast without clipping of intensity values.

\section{Western Blotting of Sperm Samples}

For western blot analysis, sperm from 3-6 males was sedimented at $3,000 \mathrm{rpm}$ for $3.5 \mathrm{~min}$. The supernatant was replaced with RIPA buffer (50 mM Tris-HCl [pH 7.5], $150 \mathrm{mM} \mathrm{NaCl}, 1 \mathrm{mM} \mathrm{MgCl}$, $1 \%$ NP-40, $0.5 \%$ sodium deoxycholate, $1 \times$ complete protease inhibitor [Roche]) including 1\% SDS. After preparation of all samples, $1 \mathrm{U} / \mu \mathrm{l}$ benzonase (Merck) was added, and samples were incubated for $30 \mathrm{~min}$ at room temperature. Samples were then mixed with $4 \times$ Laemmli buffer containing $\beta$-mercaptoethanol and boiled at $95^{\circ} \mathrm{C}$ for $5 \mathrm{~min}$. After SDS- 
PAGE, samples were wet-transferred onto a nitrocellulose membrane. Total protein was visualized by Ponceau staining before blocking with $5 \%$ milk powder in $0.1 \%$ Tween in $1 \times$ TBS (TBST). Membranes were incubated in primary mouse antizebrafish-Dcst2 antibody [1:500; (Noda et al., 2021)] overnight at $4^{\circ} \mathrm{C}$, then washed with TBST before HRP-conjugated secondary antibody [1:10.000 (115-036-062, Dianova)] incubation for $1 \mathrm{~h}$. Membranes were washed several times in TBST before HRP activity was visualized using Clarity Western ECL Substrate (BioRad) on a ChemiDoc (BioRad). For visualizing Tubulin levels, membranes were stripped using Restore Western Blot Stripping Buffer (Thermo Fisher Scientific) before washing, blocking and incubation with mouse anti-alpha-Tubulin antibody [1:20.000 (T6074, Merck)] and proceeding with secondary antibody staining and detection as described above.

To assess relative Dcst2 protein amounts, average intensities of Dcst2-specific bands were quantified in Fiji on three independent immunoblots for each genotype relative to tubulin levels, which was used as loading control. Values were then normalized to the levels of wild-type sperm.

\section{DATA AVAILABILITY STATEMENT}

The original contributions presented in the study are included in the article/Supplementary Material, further inquiries can be directed to the corresponding authors.

\section{ETHICS STATEMENT}

The animal study was reviewed and approved by the Amt der Wiener Landesregierung, Magistratsabteilung 58-Wasserrecht; Vienna, Austria.

\section{AUTHOR CONTRIBUTIONS}

$\mathrm{VD}$ and $\mathrm{AP}$ conceived the study; $\mathrm{MB}, \mathrm{AK}$, and $\mathrm{KP}$ designed, performed and analyzed experiments with contributions from

\section{REFERENCES}

Altschul, S. (1997). Gapped BLAST and PSI-BLAST: a New Generation of Protein Database Search Programs. Nucleic Acids Res. 25, 3389-3402. doi:10.1093/nar/ 25.17.3389

Barbaux, S., Ialy-Radio, C., Chalbi, M., Dybal, E., Homps-Legrand, M., do Cruzeiro, M., et al. (2020). Sperm SPACA6 Protein Is Required for Mammalian SpermEgg Adhesion/Fusion. Sci. Rep. 10, 5335. doi:10.1038/s41598-020-62091-y

Bateman, A., Martin, M.-J., Orchard, S., Magrane, M., Agivetova, R., Ahmad, S., et al. (2021). UniProt: the Universal Protein Knowledgebase in 2021. Nucleic Acids Res. 49, D480-D489. doi:10.1093/nar/gkaa1100

Bianchi, E., and Wright, G. J. (2016). Sperm Meets Egg: The Genetics of Mammalian Fertilization. Annu. Rev. Genet. 50, 93-111. doi:10.1146/ ANNUREV-GENET-121415-121834

Blum, M., Chang, H.-Y., Chuguransky, S., Grego, T., Kandasaamy, S., Mitchell, A., et al. (2021). The InterPro Protein Families and Domains Database: 20 Years on. Nucleic Acids Res. 49, D344-D354. doi:10.1093/nar/gkaa977
$\mathrm{VD}, \mathrm{AP}$, and AS performed the phylogenetic analysis; VD and AP coordinated and supervised the project; all authors contributed to writing the manuscript.

\section{FUNDING}

This research was funded by the Research Institute of Molecular Pathology (IMP), Boehringer Ingelheim, the Austrian Academy of Sciences, FFG (Headquarter grant FFG-852936), the FWF START program (Y 1031-B28) to AP, the HFSP Career Development Award (CDA00066/ 2015) and the HFSP Young Investigator Award to AP, EMBO-YIP funds to AP, and an HFSP post-doctoral fellowship to VD. The funders had no role in study design, data collection and analysis, decision to publish, or preparation of the manuscript. For the purpose of Open Access, the author has applied a CC BY public copyright license to any Author Accepted Manuscript (AAM) version arising from this submission.

\section{ACKNOWLEDGMENTS}

We thank the team of the Biooptics facility at the Vienna Biocenter, in particular Pawel Pasierbek and Thomas Lendl, for support with microscopy and image analysis; the animal facility personnel from the IMP for taking excellent care of zebrafish; Anna Bandura for help with genotyping; Juraj Ahel for providing the AlphaFold2 script; Maria Novatchkova for help with RNA sequencing analysis; Andreas Blaha and Sara Berent for valuable help with the sperm morphology assay; and the entire Pauli lab for fruitful discussions.

\section{SUPPLEMENTARY MATERIAL}

The Supplementary Material for this article can be found online at: https:/www.frontiersin.org/articles/10.3389/fcell.2021.806982/ full\#supplementary-material

Cordelieres, F. (2005). Manual Tracking. Available at: https://imagej.nih.gov/ij/ plugins/track/track.html(Accessed October 28, 2021).

Deneke, V. E., and Pauli, A. (2021). The Fertilization Enigma: How Sperm and Egg Fuse. Annu. Rev. Cel Dev. Biol. 37, 391-414. doi:10.1146/ANNUREVCELLBIO-120219-021751

Eddy, S. R. (1998). Profile Hidden Markov Models. Bioinformatics 14, 755-763. doi:10.1093/bioinformatics/14.9.755

El-Gebali, S., Mistry, J., Bateman, A., Eddy, S. R., Luciani, A., Potter, S. C., et al. (2019). The Pfam Protein Families Database in 2019. Nucleic Acids Res. 47, D427-D432. doi:10.1093/nar/gky995

Fujihara, Y., Herberg, S., Blaha, A., Panser, K., Kobayashi, K., Larasati, T., et al. (2021). The Conserved Fertility Factor SPACA4/Bouncer Has Divergent Modes of Action in Vertebrate Fertilization. Proc. Natl. Acad. Sci. USA 118, e2108777118. doi:10.1073/pnas.2108777118

Gagnon, J. A., Valen, E., Thyme, S. B., Huang, P., Ahkmetova, L., Pauli, A., et al. (2014). Efficient Mutagenesis by Cas9 Protein-Mediated Oligonucleotide Insertion and Large-Scale Assessment of Single-Guide RNAs. PLoS ONE 9 , e98186. doi:10.1371/journal.pone.0098186 
Gibson, D. G., Young, L., Chuang, R.-Y., Venter, J. C., Hutchison, C. A., and Smith, H. O. (2009). Enzymatic Assembly of DNA Molecules up to Several Hundred Kilobases. Nat. Methods 6, 343-345. doi:10.1038/ nmeth.1318

Grayson, P. (2015). Izumol and Juno: the Evolutionary Origins and Coevolution of Essential Sperm-Egg Binding Partners. R. Soc. Open Sci. 2, 150296. doi:10.1098/ rsos.150296

Hart, N. H., and Donovan, M. (1983). Fine Structure of the Chorion and Site of Sperm Entry in the Egg ofBrachydanio. J. Exp. Zool. 227, 277-296. doi:10.1002/ jez.1402270212

Herberg, S., Gert, K. R., Schleiffer, A., and Pauli, A. (2018). The Ly6/uPAR Protein Bouncer Is Necessary and Sufficient for Species-specific Fertilization. Science 361, 1029-1033. doi:10.1126/science.aat7113

Inoue, N., Ikawa, M., Isotani, A., and Okabe, M. (2005). The Immunoglobulin Superfamily Protein Izumo Is Required for Sperm to Fuse with Eggs. Nature 434, 234-238. doi:10.1038/nature03362

Inoue, N., Hamada, D., Kamikubo, H., Hirata, K., Kataoka, M., Yamamoto, M., et al. (2013). Molecular Dissection of IZUMO1, a Sperm Protein Essential for Sperm-Egg Fusion. Development (Cambridge) 140, 3221-3229. doi:10.1242/ dev.094854

Inoue, N., Hagihara, Y., and Wada, I. (2021). Evolutionarily Conserved Sperm Factors, DCST1 and DCST2, Are Required for Gamete Fusion. eLife 10, e66313. doi:10.7554/eLife.66313

Jumper, J., Evans, R., Pritzel, A., Green, T., Figurnov, M., Ronneberger, O., et al. (2021). Highly Accurate Protein Structure Prediction with AlphaFold. Nature 596, 583-589. doi:10.1038/s41586-021-03819-2

Kaji, K., Oda, S., Shikano, T., Ohnuki, T., Uematsu, Y., Sakagami, J., et al. (2000). The Gamete Fusion Process Is Defective in Eggs of Cd9-Deficient Mice. Nat. Genet. 24, 279-282. doi:10.1038/73502

Lamas-Toranzo, I., Hamze, J. G., Bianchi, E., Fernández-Fuertes, B., PérezCerezales, S., Laguna-Barraza, R., et al. (2020). TMEM95 Is a Sperm Membrane Protein Essential for Mammalian Fertilization. eLife 9, e53913. doi:10.7554/eLife.53913

le Naour, F., Rubinstein, E., Jasmin, C., Prenant, M., and Boucheix, C. (2000). Severely Reduced Female Fertility in CD9-Deficient Mice. Science 287, 319-321. doi:10.1126/science.287.5451.319

Letunic, I., and Bork, P. (2021). Interactive Tree of Life (iTOL) V5: an Online Tool for Phylogenetic Tree Display and Annotation. Nucleic Acids Res. 49, W293-W296. doi:10.1093/nar/gkab301

Miyado, K., Yamada, G., Yamada, S., Hasuwa, H., Nakamura, Y., Ryu, F., et al. (2000). Requirement of CD9 on the Egg Plasma Membrane for Fertilization. Science 287, 321-324. doi:10.1126/science.287.5451.321

Nishimura, K., Han, L., Bianchi, E., Wright, G. J., de Sanctis, D., and Jovine, L. (2016). The Structure of Sperm Izumol Reveals Unexpected Similarities with Plasmodium Invasion Proteins. Curr. Biol. 26, R661-R662. doi:10.1016/ j.cub.2016.06.028
Noda, T., Lu, Y., Fujihara, Y., Oura, S., Koyano, T., Kobayashi, S., et al. (2020) Sperm Proteins SOF1, TMEM95, and SPACA6 Are Required for Sperm-oocyte Fusion in Mice. Proc. Natl. Acad. Sci. USA 117, 11493-11502. doi:10.1073/ pnas. 1922650117

Noda, T., Blaha, A., Fujihara, Y., Gert, K., Emori, C., Deneke, V. E., et al. (2021). Sperm Membrane Proteins DCST1 and DCST2 Are Required for the SpermEgg Fusion Process in Mice and Fish. bioRxiv [Preprint]. Available at: https:// www.biorxiv.org/content/10.1101/2021.04.18.440256 (Accessed October 28, 2021).

Schoch, C. L., Ciufo, S., Domrachev, M., Hotton, C. L., Kannan, S., Khovanskaya, R., et al. (2020). NCBI Taxonomy: A Comprehensive Update on Curation, Resources and Tools, Database 2020, 1-21. doi:10.1093/database/baaa062

Sievers, F., Wilm, A., Dineen, D., Gibson, T. J., Karplus, K., Li, W., et al. (2011). Fast, Scalable Generation of High-Quality Protein Multiple Sequence Alignments Using Clustal Omega, Mol. Syst. Biol. 7, 539. doi:10.1038/ msb. 2011.75

Stein, P., Savy, V., Williams, A. M., and Williams, C. J. (2020). Modulators of Calcium Signalling at Fertilization. Open Biol. 10, 200118. doi:10.1098/ RSOB. 200118

Waterhouse, A. M., Procter, J. B., Martin, D. M. A., Clamp, M., and Barton, G. J. (2009). Jalview Version 2--a Multiple Sequence Alignment Editor and Analysis Workbench. Bioinformatics 25, 1189-1191. doi:10.1093/bioinformatics/btp033

Wolenski, J. S., and Hart, N. H. (1987). Scanning Electron Microscope Studies of Sperm Incorporation into the Zebrafish (Brachydanio) Egg. J. Exp. Zool. 243, 259-273. doi:10.1002/jez.1402430211

Yanagimachi, R., Cherr, G., Matsubara, T., Andoh, T., Harumi, T., Vines, C., et al. (2013). Sperm Attractant in the Micropyle Region of Fish and Insect Eggs1. Biol. Reprod. 88, 47. doi:10.1095/biolreprod.112.105072

Conflict of Interest: The authors declare that the research was conducted in the absence of any commercial or financial relationships that could be construed as a potential conflict of interest.

Publisher's Note: All claims expressed in this article are solely those of the authors and do not necessarily represent those of their affiliated organizations, or those of the publisher, the editors and the reviewers. Any product that may be evaluated in this article, or claim that may be made by its manufacturer, is not guaranteed or endorsed by the publisher.

Copyright (c) 2022 Binner, Kogan, Panser, Schleiffer, Deneke and Pauli. This is an open-access article distributed under the terms of the Creative Commons Attribution License (CC BY). The use, distribution or reproduction in other forums is permitted, provided the original author(s) and the copyright owner(s) are credited and that the original publication in this journal is cited, in accordance with accepted academic practice. No use, distribution or reproduction is permitted which does not comply with these terms. 